\title{
Utility of 4-(4-Bromophenyl)-4-oxo-but-2-enoic Acid in Synthesis of Some Important Heterocyclic Compounds
}

\author{
S.A. Rizk*, M.A. El-Hashash, Sohir A. Shaker and K.K. \\ Mostafa \\ Chemistry Department, Faculty of Science, Ain Shams \\ University, Cairo, Egypt.
}

\begin{abstract}
7 HE PRESENT work deals with the generation and synthesis of different heterocycles via the treatment of 3-(4-bromobenzoyl) prop-2-enoic acid (1) with thiourea, ethylcyanoacetate malononitrile \& acetylacetone in presence of amm.acetate and / or piperidine , 2amino-5-phthalimidomethy 11,3,4-thiadiazole, methyl thioglycolate 4bromoaniline and ethylacetoacetate to afford Michael and azaMichael adduct that cyclized by hydroxyl amine and hydrazine hydrate, respectively. Additionally, utility of 2-(2-amino-5phthalimidomethyl 1,3,4-thiadiazol-2-yl)-4-(4-bromophenyl)-4-oxobutanoic acid (4) as a key starting material to synthesize some important heterocycles include fused oxoimidazolo [2,3-b]-1,3,4thiadiazole.
\end{abstract}

Keywords: 4-Bromophenyl-4-oxo-2-butenoic acid, Pyridine, Pyrimidine , Phthalazinhydrazone, Benzisoxazolone, Phthalimide, Thiadiazole imidazolothiadiazole and Pyridazinone.

It has been reported ${ }^{(1,2)}$ that 3-aroylacrylic acids were used as inhibitors of phospholipase from snake venom and from procaine pancreas, also they have antibacterial activities ${ }^{(3,4)}$, which prevent the growth of Staphylococcus aureus, beside their anti-proliferative ${ }^{(5)}$ action against human cervix carcinoma. Recently $^{(6,7)} 3$-(4-acetamido) and \or 4-chloro benzoyl prop-2-enoic acid were used in the synthesis of some heterocyclic compounds. Hence, keeping these reports in view and continuation of our search for 3-aroyl prop-2-enoic acid derivatives $^{(8-13)}$, the present work deals with the study of the behaviour of 3-(4bromobenzoyl)prop-2-enoic acid towards the action of different nucleophilic species including carbon, nitrogen nucleophiles and the utility some of the reaction products in heterocyclic synthesis, hoping to get new compounds of anticipated biological activities.

\section{Results and Discussion}

Addition of ethyl cyanoacetate on 3-(4-bromobenzoyl)-acrylic acid 1 in the presence of ammonium acetate yielded a mixture of ethyl-2-amino-4-carboxy-6(4-bromophenyl)-nicotinate 2 and 3-cyano-4-carboxy-6-(4-bromophenyl)-2(1H)-

*E-mail: samehrizk2006@gmail.com 
pyridones 3, whereas compounds 2 and 3 can be explained ${ }^{(14)}$. Also, in the present investigation, similar treatment of 1 with malononitrile in the presence of ammonium acetate in boiling butanol gave 2-amino-3-cyano-4-carboxy-6(4bromo)phenyl 3,4-dihydropyridine 4 . The structure of $1: 1$ adduct 4 obtained by base catalyzed Michael addition of malononitrile to acid 1 is elucidated by studying their spectroscopic properties. The mass spectrum of 4 shows the correct molecular ion peak. On the other hand, when compound 1 was treated with malononitrile in the presence of piperidine in boiling ethanol yielded 2amino-3-cyano-4-carboxy-6-(4-bromophenyl)-pyrane (5) (Scheme 1).

The pyrimidines and their ring-fused derivatives are one of the most active classes of compounds, possessing a wide spectrum of biological activity ${ }^{(15)}$. They are known as heterocyclic core of the nucleic acid bases. These ring systems are often incorporated into drugs designed for anticancer ${ }^{(16,17)}$, antiviral ${ }^{(18)}$, antihypertensive $^{(19)}$, analgesic ${ }^{(20)}$, antipyretic ${ }^{(21)}$, anti-inflammatory ${ }^{(22)}$, antipsoriasis $^{(23)}$ agents. Some of them are active on the blood circulatory system ${ }^{(24)}$ and can stimulate the skin regeneration and increase the efficacy of antibiotic therapy of Staphylococcus and Proteus infected wounds ${ }^{(25)}$. The authors sought to investigate the behavior of 1 with thiourea in boiling ethanol in the presence of sodium ethoxide under Michael reaction condition afforded 4-(4-bromophenyl)6-carboxypyrimidin-2 $(1 \mathrm{H})$ thione (6) (Scheme 1). The structure of compound 6 is confirmed by correct microanalytical data and also by spectral evidence. $v \mathrm{C}=\mathrm{O}$ at 1676. The lower absorption for carbonyl group is due to formation of conjugated double bond results from dehydrogenation, i.e., formation of $\alpha, \beta$ unsaturated system. The EI-MS shows the molecular ion peak at m/e 312 and 310 corresponding to $(\mathrm{M}+2)^{\cdot+}\left(\mathrm{M}^{++}\right)$, respectively.

Morever, Reaction of 3-(4-bromo) benzoyl acrylic acid (1) with acetylactone in refluxing methanol in the presence of sodium methoxide (Michael condition) afforded 3-(4-bromophenyl)-5-carboxy-6-acetylcyclohexen-1-one (7). This substituted cyclohexenone derivative 7 is considered as a key starting material for diverse of some interesting heterocyclic compounds. The structure of compound 7 is deduced from its micro analytical and spectral data. The presence of the intramolecular hydrogen bond (chelated form) causes lowering $v \mathrm{C}=\mathrm{O}$ as expected. Further support for the proposed structure of 7 was gained from the EI-MS spectrum that the $\mathrm{m} / \mathrm{e} 320,318\left(\mathrm{M}+2, \mathrm{M}^{+}-\mathrm{H}_{2} \mathrm{O}\right)$. The derivatives of naphthoheterocyclic have attracted the attention of many organic chemists owing to their well pronounced activities such as anticancer ${ }^{(26)}$, antifungal, cytotoxic ${ }^{(27)}$ and in the treatment of metabolic disorders ${ }^{(28)}$. Reaction of acetylcyclohexenone derivative 7 with hydrazine hydrate in boiling ethanol yielded $1(2 \mathrm{H})$ phthalazinone derivative 8 . Condensation takes place firstly with reactive acetyl carbonyl followed by ring closure then formation of the corresponding hydrazone. The structure of compound 8 is substantiated by spectroscopic tools. In EI-MS exhibits molecular ion peaks m/e (347, 349 18.2\%) beside some of abundant peaks.

Egypt. J. Chem. 55, No.1 (2012) 
Reaction of 7 with hydroxylamine hydrochloride in boiling pyridine gave the benzisoxazole derivative 9 and not $4 \mathrm{H}-2,3$-benzoxazin-4-one 10 which is less thermodynamically stable than 9 . The structure of compound 9 is confirmed by correct micro analytical data and also by spectral e. Addition of methyl sulfahydrylacetate on 3-(4-bromobenzoyl)-acrylic acid (1) in the presence of few drops of piperidine yielded a mixture of 2-methoxy carbonyl methmercapto-4-(4bromophenyl)-4-oxo-butanoic acid (11) and 2,6-dioxo-3-(4-bromoacetophenyl)2,3,5,6-tetrahydro-1,4-oxathiine (12), whereas compounds 11 and 12 can be explained, thia-Michael adducts followed by ring closure afford product 12 . Also, in the present investigation, similar treatment of 1 with 2-amino-5-phthalimidomethyl1,3,4-thiadiazole in boiling ethanol gave 2-([5-phthalimid-omethyl]-1,3,5-thiadiazol2-yl)amino-4-oxo-4-(4-bromophenyl) butanoic acid (13). The structure of 1:1 adduct 13 is obtained by aza- Michael addition of 2-amino thiadiazole to acid 1 that elucidated chemically (Scheme 2 ) and by studying their spectroscopic properties. The mass spectrum of 13 shows the correct molecular ion peak. On the other hand,when compound 1 was treated with 4-bromo aniline in the presence of piperidine in boiling ethanol yielded 2-(4-bromophenyl)amino-4-(4-bromophenyl)-4oxo-butanoic acid (14) (Scheme 2). Refluxing of 13 with freshly distillated acetic anhydride afforded 2-phthalimidomethyl-4-oxo-5-(4-bromobenz-oylmethyl) imidazolo [2,1-b]-1,3,4-thiadiazole 15(Scheme 2). Its IR displayed an absorption bands at $1772,1720,1691,1668 \mathrm{~cm}-1$ attributable to $v \mathrm{C}=\mathrm{O}$ and showed no absorption frequency in the NH region. The H-NMR spectrum of compound 15 showed singlet peak at 6.7 corresponding to bridged $\mathrm{CH}, 1,3$-double bond shift that explained the proton spend apart of life time as methine proton. The $3(2 \mathrm{H})$ pyridazinones and their ring-fused derivatives are one of the most active classes in drug discovery, with many of their analogs being in the treatment of various human pathological states.They were described as nonsteroidal anti-inflamatory drugs, e.g. Emorfazone and related compounds ${ }^{(29)}$ agents for therapeutic intervention of renal urologic, e.g. FK-838 ${ }^{(30)}$, cardiovascular, e.g. EMD-57283 ${ }^{(31)}$, respiratory, e.g. NIP$502^{(32)}$ and deramatologic diseases, e.g. FR- $1818177^{(33)}$, pyridazinone PDE inhibitors developed from ibudilast ${ }^{(34)}$. Thus, when acids 13,14 were allowed to react with hydrazine hydrate in boiling ethanol ${ }^{(13-16)}$ afforded 16,17 , respectively. The structure of compounds 16, 17 is confirmed by correct microanalytical data and also by spectral evidence. $\mathrm{vC}=\mathrm{O}$ at 1676 . The lower absorption for carbonyl group is due to formation of lactam-lactim dynamic equilibrium in pyridazinone moiety, i.e., formation of amide system. The EI-MS for 17 shows the molecular ion peak at $\mathrm{m} / \mathrm{e}$ 250 corresponding to ( $\mathrm{M}^{+}-4$-bromoaniline) evidence. Refluxing compound 16 with ethyl chloro acetate in the presence of anhydrous $\mathrm{K} 2 \mathrm{CO} 3$ afforded 2-(ethoxy carbonyl methyl-4-(5-phthalimidomethyl-1,3,4-thiadiazol-2-yl) amino-6-(4-bromophenyl) 4,5-dihydro-pyridazinone(18). The reaction takes place via SN2 mechanism to give the desired products 18 , respectively. Structure of compound 18 was inferred 
from microanalytical and spectral data. Its IR spectrum revealed strong absorption bands at $3300,3160,1770,1745,1691,1680 \mathrm{~cm}^{-1}$ attributable to $v \mathrm{NH}, v \mathrm{C}=\mathrm{O}$ of ester phthalimido and amide groups. ${ }^{1} \mathrm{HNMR}$ DMSO exhibits signals at $1.5(\mathrm{t}, 3 \mathrm{H}, \mathrm{CH} 3)$, $4.2(\mathrm{q}, 2 \mathrm{H}, \mathrm{OCH} 2)$ and $5(\mathrm{~s}, 2 \mathrm{H}, \mathrm{NCH} 2 \mathrm{CO}), 5.5$ in side to $3.0\left(2 \mathrm{dd}, \mathrm{CH}_{2-} \mathrm{C}=\mathrm{O} \mathrm{J}=7.7\right)$ (diastereotopic protons), 3.8 (dd, $\mathrm{CH}-\mathrm{COOH}$, methineproton) (s, 2H, $\mathrm{CH} 2), 6.5$ (s, $\mathrm{NH})$, 7.4-7.8 (m, 8H, ArH) ArH) that confirmed with this structure.

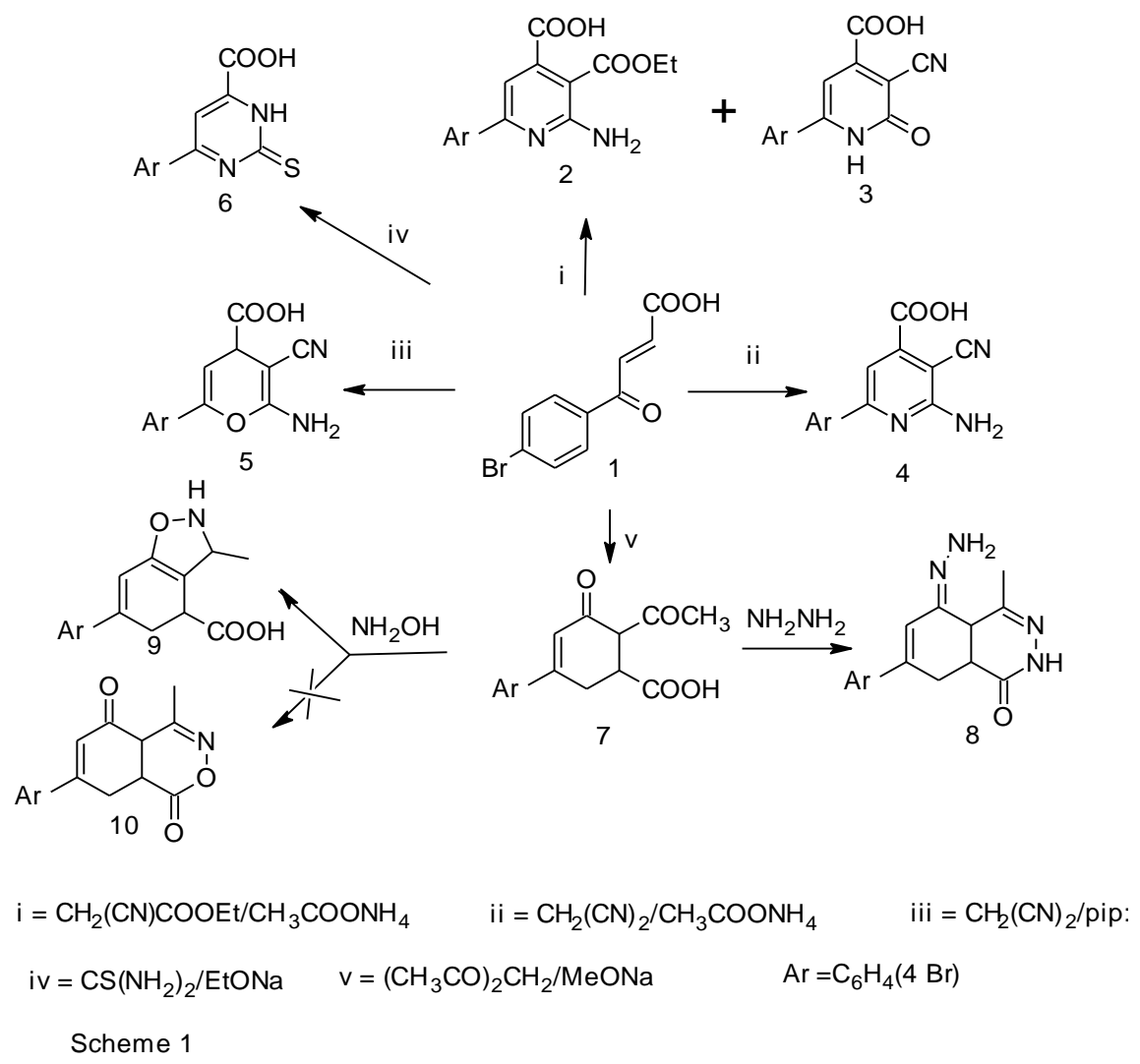

Scheme 1.

Egypt. J. Chem. 55, No.1 (2012) 


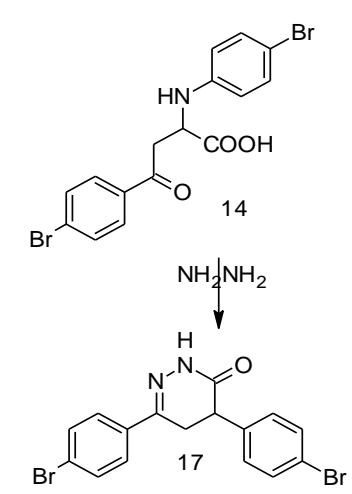<smiles>CC(=O)CC(CC(=O)c1ccc(Br)cc1)CC(=O)c1ccc(Br)cc1</smiles><smiles></smiles>

15
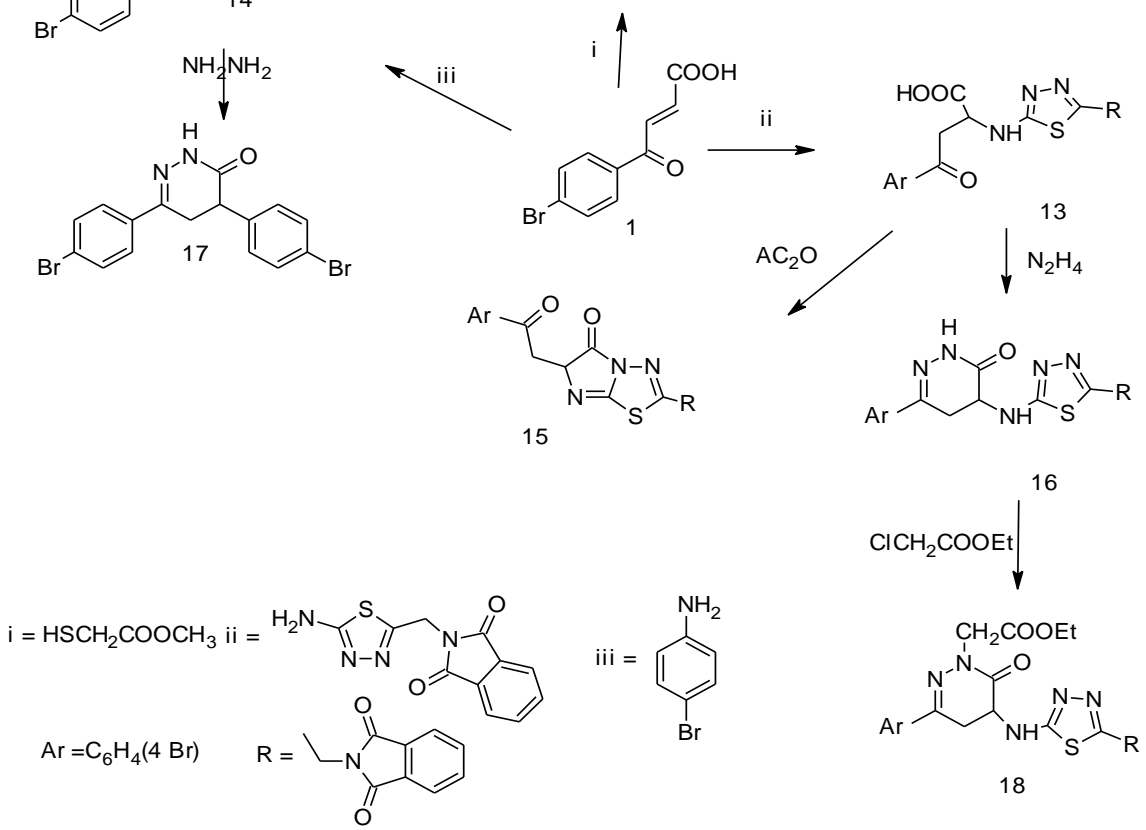

Scheme 2

Scheme 2.

\section{Conclusion}

The proposed procedure is an easy and inexpensive methodology for the synthesized compounds. Some new Some interesting heterocycles were synthesized by the reaction of 3-(4-bromo benzoyl)prop-2-enoic acid precursors with ethyl cyano acetate,malononitrile in different condition,acetyl acetone under Michael reaction condition ethyl thioglycolate, 5-phthalimidomethyl-2-amino1,3,4-thiadiazole and, 3-bromo aniline followed by cyclization within binucleophiles $\mathrm{NH} 2 \mathrm{NH} 2$ and $\mathrm{NH} 2 \mathrm{OH}$. Synthesis a various substituted pyridazinones derivatives incorporated with 1,3,4-thiadiazole 14,16 and imidazolo [2,1-b]-1,3,4-thiadiazole 11. 


\section{Experimental}

All melting points are uncorrected. Elemental analyses were carried out in the Microanalytical Center, the Center Publication for Research, Cairo, Egypt. By Elementar Viro El Microanalysis IR spectra $(\mathrm{KBr})$ were recorded on infrared spectrometer ST-IR DOMEM Hartman Braun, Model: MBB 157, Canada and HNMR spectra recorded on a varian $300 \mathrm{MHz}$ (Germany 1999) using TMS as internal standard. The mass spectra were recorded on Shimadzu GCMS-QP-1000 EX mass spectrometer at 70e.v. homogeneity of all compounds synthesized was checked by TLC.

Ethyl-2-amino-4-carboxy-6-(4-bromo phenyl) nicotinate (2)

A solution of $1(2.5 \mathrm{~g}, 0.01 \mathrm{~mol}), 3 \mathrm{ml}$ ethylcyanoacetate and $5 \mathrm{~g}$ ammonium acetate was heated in water bath for $3 \mathrm{hr}$, then poured water, the solid that separated with crystallized form ethanol to afford 2. M.wt $365\left(\mathrm{C}_{15} \mathrm{H}_{13} \mathrm{BrN} 2 \mathrm{O}_{4}\right)$ (m.p. $115^{\circ} \mathrm{C}$, yield 54\%,\% calcd/found [(C 49.30/49.22, \% $\mathrm{H} 3.5 / 3.46, \% \mathrm{~N}$ 7.67/7.66, \% $\mathrm{Br} 21.9 / 21.6]$. IR vNH.3437, vC $=\mathrm{O}$ (acid and ester)1686, 1733, $v \mathrm{C}=\mathrm{N} 1620 \mathrm{~cm}^{-1}{ }^{1} \mathrm{HNMR} 1.3(\mathrm{t}, 3 \mathrm{H}, \mathrm{J}=7.4)$, 3.9(s,2H,NH2), $4.05(\mathrm{q}, 2 \mathrm{H}, \mathrm{J}=7.4)$, 7.57.8 (m,5H,ArH),11.1(s,1H,COOH). The EI-MS shows the molecular ion peak at $\mathrm{m} / \mathrm{e} 366$ and 364 corresponding to $(\mathrm{M}+2)^{.^{+}}\left(\mathrm{M}^{+}\right)$, respectively .

3-Cyano-4-carboxy-6-(4-bromo phenyl) 2(1H)-pyridones (3)

A solution of 1 ( $2.5 \mathrm{~g}, 0.01 \mathrm{~mol}), 3 \mathrm{ml}$ ethylcyanoacetate and $5 \mathrm{~g}$ ammonium acetate was heated in water bath for $3 \mathrm{hr}$, then poured water, the solid that separated was crystallized form Benzene - Ethanol to afford 3.M.wt $=319\left(\mathrm{C}_{13} \mathrm{H}_{7}\right.$ $\mathrm{Br} \mathrm{N}_{2} \mathrm{O}_{3}$ ) (m.p $160^{\circ} \mathrm{C}$, yield $40 \%, \%$ calcd/found [ $(\mathrm{C} 48.92 / 48.94, \mathrm{H} \mathrm{2.19/2.18, \textrm {N }}$ $8.78 / 8.81$, Br 25.07/25.11 IR vNH 3354, vCN 2212, v max of two carbonyl groups (cyclic amide and carboxyl group), 1655, 1678, and vC=N1628, ${ }^{1} \mathrm{HNMR}$ $6.8-7.5(\mathrm{~m}, 5 \mathrm{H}, \mathrm{ArH}), 10.03(\mathrm{~s}, 1 \mathrm{H}, \mathrm{NH}) 12.1(\mathrm{~s}, 1 \mathrm{H}, \mathrm{COOH})$. The EI-MS shows the molecular ion peak at $\mathrm{m} / \mathrm{e} 320$ and 318 corresponding to $(\mathrm{M}+2)^{+^{+}}\left(\mathbf{M}^{+}\right)$, respectively.

2-Amino-3-cyano-4-carboxy-6-(4-bromophenyl)-3,4-dihydropyridine (4)

A solution of $1(2,5 \mathrm{~g}, 0.01 \mathrm{~mol})$ in n-butanol $(20 \mathrm{ml})$ was treated with malononitrile ( $0.7 \mathrm{~g}, 0.01$ mole) in $5 \mathrm{~g}$ ammonium acetate refluxed for $3 \mathrm{hr}$, then poured water with heating to replace $n$-butanol by water, then take the filtrate with ice $/ \mathrm{HCl}$. The solid that separated on cooling was crystallized form ethanol to afford 4. M. wt=320 $\left(\mathrm{C}_{13} \mathrm{H}_{10-} \mathrm{BrN}_{3} \mathrm{O}_{2}\right)$ m.p. $220{ }^{\circ} \mathrm{C}$, yield $75 \%$ calcd $/$ found [(C48. 75/49.00 , H 3.13/3.22 , N 13.12/13.02 , Br25.00/25.08] IR vOH, vNH, vCN, vC $=\mathrm{O}$ at $3422,3220,2211,1707 \mathrm{~cm}^{-1}{ }^{1} \mathrm{HNMR} \quad 2.4(\mathrm{~s}, 2 \mathrm{H}, \mathrm{NH} 2), 2.8$ $(\mathrm{d}, 1 \mathrm{H}, \mathrm{CHCN}, \mathrm{J}=8.5), 3.2(\mathrm{dd}, \mathrm{H}, \mathrm{CHCO} .5, \mathrm{~J}=8.5, \mathrm{~J}=6.4), 5.6(\mathrm{~d}, 1 \mathrm{H}, \mathrm{H}-5 \mathrm{pyr}, \mathrm{J}=$ 6.4) $7.4-7.5(\mathrm{~m}, 4 \mathrm{H}, \mathrm{Ar}-\mathrm{H}), 11.03(\mathrm{~s}, 1 \mathrm{H}$, exchangeable proton. The EI-MS shows the molecular ion peak at $\mathrm{m} / \mathrm{e} 321$ and 319 corresponding to $(\mathrm{M}+2)^{+{ }^{+}}\left(\mathrm{M}^{+}\right)$, respectively. 


\section{2-Amino-3-cyano-4-carboxy-6-(4-bromophenyl )pyran(5)}

A solution of $1(2.5 \mathrm{~g}, 0.01 \mathrm{~mol})$ in ethanol $(100 \mathrm{ml})$ was treated with malononitrile $(0.7 \mathrm{~g}, 0.01 \mathrm{~mol})$ in piperidine $(2 \mathrm{ml})$, stirred at room temperature for $1 \mathrm{hr}$, then concentrated the solution and poured $\mathrm{H}_{2} \mathrm{O} / \mathrm{HCL}$. The solid that separated on cooling was crystallized form ethanol to afford 5. M.wt $=321$ $\left(\mathrm{C}_{13} \mathrm{H}_{9} \mathrm{Br} \mathrm{N} \mathrm{N}_{2} \mathrm{O}_{3}\right)$ (m.p $121^{\circ} \mathrm{C}$, yield $70 \%$, \% calcd/found [(C 48.59/48.48, $\mathrm{H}$ 2.80/2.77, N 8.72/8.68, Br 24.92/25.81 IR vNH (bonded and non bonded), 3227, $2339, \mathrm{C}=\mathrm{O} 1705 \mathrm{~cm}^{-1}$ and $v \mathrm{C}=\mathrm{N} 1625,{ }^{1} \mathrm{HNMR} 2.6(\mathrm{~s}, 2 \mathrm{H}, \mathrm{NH} 2), 2.8(\mathrm{~d}, 1 \mathrm{H}, \mathrm{CH}-\mathrm{CN})$, $3,2(\mathrm{~d}, 1 \mathrm{H}, \mathrm{CH}-\mathrm{CO}), 7 .-7.5(\mathrm{~m}, 5 \mathrm{H}, \mathrm{Ar}-\mathrm{H}), 11.03(\mathrm{~s}, 1 \mathrm{H}$,exchangeable proton). The EI-MS shows the molecular ion peak at $\mathrm{m} / \mathrm{e} 322$ and 320 corresponding to $(\mathrm{M}+2)^{++}\left(\mathrm{M}^{+}\right)$, respectively.

4-(4-Bromophenyl)-6-carboxy pyrimidin-2(1H)-thione (6)

A solution of $1(2.5 \mathrm{~g}, 0.01 \mathrm{~mol})$ in $0.5 \mathrm{~g}$ sodium and $15 \mathrm{ml}$ ethanol was treated with thiourea $(0,76 \mathrm{~g}, 0.01 \mathrm{~mol})$ and refluxed for $4 \mathrm{hr}$. The solid that separated after cooling was crystallized form the suitable solvent to afford 6 . M.wt $=311\left(\mathrm{C}_{11} \mathrm{H}_{7} \mathrm{Br} \mathrm{N}_{2} \mathrm{O}_{2} \mathrm{~S}\right)$ (m.p $200^{\circ} \mathrm{C}$,yield $75 \%$, \% calcd/found $[(\mathrm{C}$ 42.44/42.48, H 2.25/2.35,N 8.99/8.98,S 10.28 /10.23, Br 25.72/25.61. IR vOH and/or $v \mathrm{NH} v 3379,3275,3180$ and $v \mathrm{C}=\mathrm{O} 1676 \mathrm{vC}=\mathrm{N}$ at $1613 \mathrm{~cm}^{-1},{ }^{1} \mathrm{HNMR}$ $3.9(\mathrm{~s}, 1 \mathrm{H}, \mathrm{NH}), 6.4(\mathrm{~s}, 1 \mathrm{H}$, pyrimidine proton $), 7.4(\mathrm{~d}, 4 \mathrm{H} . \mathrm{Ar}-\mathrm{H}), 11(\mathrm{~s}, 1 \mathrm{H}, \mathrm{COOH})$. The EI-MS shows the molecular ion peak at $\mathrm{m} / \mathrm{e} 312$ and 310 corresponding to $(\mathrm{M}+2)^{++}\left(\mathrm{M}^{++}\right)$, respectively .

\section{3-(4-Bromophenyl)-5-carboxy-6-acetylcyclohexen-1-one (7)}

A solution of $1(2.5 \mathrm{~g}, 0.01 \mathrm{~mole})$ in $30 \mathrm{ml}$ ethanol was treated with acetyl acetone $(0.01$ mole) refluxed in water bath for $4 \mathrm{hr}$, then poured water. The solid that separated on cooling was crystallized form the suitable solvent to afford 7 . M.wt $=337\left(\mathrm{C}_{15} \mathrm{H}_{13} \quad \mathrm{Br} \quad \mathrm{O}_{4}\right) \quad\left(\mathrm{m} \cdot \mathrm{p} \quad 119^{\circ} \mathrm{C}\right.$, yield $75 \%$, \% calcd/found $[(\mathrm{C} 53.41 / 53.60, \mathrm{H} 3.85 / 4.00, \mathrm{Br} 23.72 / 23.72$. IR exhibits $v \mathrm{OH}=3430, v \mathrm{CH}$ ar $=$ $3030 v \mathrm{CH}$ ali $=2888, v \mathrm{C}=\mathrm{O} 1687,1695 \mathrm{~cm}^{-1},{ }^{1} \mathrm{HNMR} 2.2(\mathrm{~s}, 3 \mathrm{H} . \mathrm{CH} 3 \mathrm{CO}), 2.3(\mathrm{dd}$, $2 \mathrm{H}$, diasteriotopic protons of allylic cyclohexanone), $2.9(\mathrm{~m}, 1 \mathrm{H}, \mathrm{CH}-\mathrm{COO}), 3.6(\mathrm{~d}$ $\mathrm{d}, 1 \mathrm{H}, \mathrm{CH}-\mathrm{CO}), \quad 6.8-7.3(\mathrm{~m}, 5 \mathrm{H}, \mathrm{Ar}-\mathrm{H}), 11.3(\mathrm{~s}, 1 \mathrm{H}, \mathrm{COOH})$. The EI-MS spectrum shows that the $\mathrm{m} / \mathrm{e} 319,317\left(\mathrm{M}^{+}-\mathrm{H}_{2} \mathrm{O}\right)$.

1-Methyl 4,5-dihydro-6-(4-bromo phenyl) 8-hydrazino phthalazin-4(3H)-one (8)

A solution of $7(3.4 \mathrm{~g}, 0.01 \mathrm{~mol})$ in $50 \mathrm{ml}$ ethanol was treated with hydrazine hydrae $(0.01$ mole) refluxed in for $3 \mathrm{hr}$, then heated to concentrate. The solid that separated after cooling was crystallized form the suitable solvent to afford 8 . M.wt $=347\left(\mathrm{C}_{15} \mathrm{H}_{15} \quad \mathrm{Br} \mathrm{N}_{4} \mathrm{O} \quad\right.$ (m.p $280^{\circ} \mathrm{C}$, yield $55 \%$ \% calcd/found $[(\mathrm{C}$ 51.87/51.60, H 4.32/4.30, N 16.13/16.11 Br 23.05/23. 12. IR (vN H, 32003262 bonded and nonbonded $v \mathrm{C}=\mathrm{O} 1657 \mathrm{~cm}^{-1} .{ }^{1} \mathrm{HNMR} \quad 0.9$ (S,3H,CH3), 2.1 (s, $2 \mathrm{H}, \mathrm{N}=\mathrm{NH} 2), 2.5(\mathrm{~d}, 2 \mathrm{H}$, allylic, $\mathrm{J}=8.7$ ), $3.1(\mathrm{dt}, 1 \mathrm{Hb}$, fused ring, $\mathrm{J}=8.7, \mathrm{~J}=9.2), 3.5$ $(\mathrm{d}, 1 \mathrm{Ha}$,fused-ring $\mathrm{J}=9.2), 6.4(\mathrm{~s}, 1 \mathrm{H}$,olefin proton),7.27.4 (dd,4H,ArH), $11(\mathrm{~s}, 1 \mathrm{H}, \mathrm{NH})$. EI-MS exhibits molecular ion peak $\mathrm{m} / \mathrm{e}(348,18.2 \%)$ beside some of abundant peaks. 
3- Methyl 6(4-bromo phenyl)2,3-dihydro-1,2-benzoxazole-4-carboxylic acid (9)

A solution of $7(3.4 \mathrm{~g}, 0.01 \mathrm{~mol})$ in $20 \mathrm{ml}$ pyridine was treated with hydroxylamine $\left(0.01\right.$ mole) refluxed for $3 \mathrm{hr}$, then poured ice $/ \mathrm{H}_{2} \mathrm{O}$. The solid that separated after cooling was crystallized form Benzene to afford 9. M.wt = $336\left(\mathrm{C}_{15} \mathrm{H}_{14} \mathrm{Br} \mathrm{NO}_{3}\right.$ (m.p $166^{\circ} \mathrm{C}$, yield 55\% , \% calcd/found [(C 53.57/53.60, $\mathrm{H}$ 4.16/4.20, N 4.16/4.21 Br 23.81/23.77 IR (vOH 3250 (saturatedacid)., vC=O, $1700 v, v \mathrm{C}=\mathrm{N} 1618 \mathrm{~cm} .{ }^{1} \mathrm{HNMR} 1.2(\mathrm{~d}, 3 \mathrm{H} . \mathrm{CH} 3, \mathrm{~J}=7.5) 2.1(\mathrm{~s}, 2)$.

\section{Compounds 11 and 12}

A solution of $1(2.5 \mathrm{~g}, 0.01 \mathrm{~mol})$, ethylthioglycolate $(0.01 \mathrm{~mol}, 1.3 \mathrm{ml})$ and few drops of piperidine in boiling benzene was heated under reflux for $4 \mathrm{hr}$, the solid that separated after concentration was crystallized from benzene to afford 12 and ethanol to afford 11 .

2-Methoxy carbonyl methmercapto-4-(4-bromophenyl)-4-oxo-butanoic acid (11)

M.wt $361\left(\mathrm{C}_{13} \mathrm{H}_{13} \mathrm{BrSO}_{5}\right)$ (m.p. $173^{\circ} \mathrm{C}$, yield $50 \%$, \% calcd/found [(C 43.23/43.22, \%H 3.62/3.46, \% S 8.88/8.66, \% Br 22.13/22.26]. IR vOH.3437, $v \mathrm{C}=\mathrm{O}$ (acid and ester) $1678,1705,1738, v \mathrm{C}=\mathrm{N} 1620 \mathrm{~cm}^{-1}{ }^{1} \mathrm{HNMR} \delta$ 2.81(2dd,1 $\mathrm{Ha},(\mathrm{J}=15.2, \mathrm{~J}=7.2)$ and $1 \mathrm{Hb}$ methylene protons, $\mathrm{CH}_{2}-\mathrm{C}=\mathrm{O},(\mathrm{J}=15.2$, $\mathrm{J}=5.1$ ) diastereotopic protons), 3,2 (dd, $\mathrm{CH}-\mathrm{COO}$, sterogenic methine proton , $\mathrm{J}=7.2, \mathrm{~J}=5.1), 3.3(\mathrm{~s}, 2 \mathrm{H}, \mathrm{CH} 2), 3.80(\mathrm{~s}, 3 \mathrm{H}, \mathrm{CH} 3)$, multiplet at $7.47-7.75$ assigned for $4 \mathrm{ArH}$ aromatic protons, singlet $11.1(\mathrm{~s}, 1 \mathrm{H}, \mathrm{COOH}$, a acidic proton which exchanged in D2O). The EI-MS shows the molecular ion peak at $\mathrm{m} / \mathrm{e} 362$ and 360 corresponding to $(\mathrm{M}+2)^{+}\left(\mathrm{M}^{+}\right)$, respectively.

2,6-Dioxo -3-(4-bromoacetophenyl)-2,3,5,6-tetrahydro-1,4-oxathiine (12)

M.wt $=329\left(\mathrm{C}_{12} \mathrm{H}_{9} \mathrm{Br} \mathrm{SO}_{4}\right)\left(\mathrm{m} . \mathrm{p} 130^{\circ} \mathrm{C}\right.$, yield $35 \%$, \% calcd/found $[(\mathrm{C}$ 43.77/43.94, H 2.73/2.58, S 9.73/9.81, Br 24.31/24.41. IR vmax of two carbonyl groups (cyclic anhydride), $1805,1728,1680 v \mathrm{C}=\mathrm{O}$ and $v \mathrm{C}=\mathrm{N} 1628,{ }^{1} \mathrm{HNMR} \delta$ $2.80\left(2 \mathrm{dd}, 1 \mathrm{Ha},(\mathrm{J}=15.2, \mathrm{~J}=7.2)\right.$ and $1 \mathrm{Hb}$ methylene protons , $\mathrm{CH}_{2}-\mathrm{C}=\mathrm{O},(\mathrm{J}=15.2$, $\mathrm{J}=5.1$ ) diastereotopic protons), 3, 30 (dd, $\mathrm{CH}-\mathrm{COO}$, sterogenic methine proton , $\mathrm{J}=7.2, \mathrm{~J}=5.1), 3.5(\mathrm{~s}, 2 \mathrm{H}, \mathrm{CH} 2)$, multiplet at 7.50-7.70 assigned for 4ArH aromatic protons, singlet . The EI-MS shows the molecular ion peak at $\mathrm{m} / \mathrm{e} 330$ and 328 corresponding to $(\mathrm{M}+2)^{++}\left(\mathrm{M}^{+}\right)$, respectively .

2-([5-Phthalimidomethyl]-1,3,5-thiadiazol-2-yl) amino-4-oxo-4-(4-bromophenyl) butanoic acid (13)

A solution of 3-(4-bromobenzoyl)-prop-2-enoic acid (2.55 g; $0.01 \mathrm{~mol})$ and 5phthalimido methyl-2-amino 1,3,4-thiadiazole $(4.2 \mathrm{~g} ; 0.016 \mathrm{~mol})$ in $30 \mathrm{ml}$ ethanol was refluxed for $3 \mathrm{hr}$. The crude product was washed by petroleum ether (b.p $\left.40-60^{\circ} \mathrm{C}\right)$, and then, crystallized from ethanol. M.wt $=515\left(\mathrm{C}_{21} \mathrm{H}_{15} \mathrm{BrSN}_{4} \mathrm{O}_{5}\right)$ m.p. $220{ }^{\circ} \mathrm{C}$, yield $75 \%$ calcd/found $[(\mathrm{C} 48.93 / 49.00, \quad \mathrm{H}$ 2.91/3.22, $\mathrm{N}$ 10.87/10.62, Br 15.53/15.08 S 6.22/6.19.IR vOH, vNH, $v \mathrm{C}=\mathrm{O}$ at 3442,3220 , $1770,1715,1690,1680 \mathrm{~cm}^{-1} .{ }^{1} \mathrm{HNMR}$ DMSO exhibits signals at 3.4 (oct, $\mathrm{CH}_{2}$ $\mathrm{C}=\mathrm{O} \mathrm{J}=7.7$ ) (diastereotopic protons) adjacent to ketonic group are non equivalent and each proton appears as douplet (4 lines,dd), each line couples with methine

Egypt. J. Chem. 55, No.1 (2012) 
proton and gives two douplet of douplets (8 lines, $2 \mathrm{dd})$ ), 4.2 (dd,CH-COOH, methineproton) 5.5 (s,2H,CH2), $6.7(\mathrm{~s}, \mathrm{NH}), \quad 7.4-7.8(\mathrm{~m}, 8 \mathrm{H}, \mathrm{ArH}) \mathrm{ArH}), 9.2$ $(\mathrm{s}, 1 \mathrm{H}, \mathrm{COOH})$. The EI-MS shows the molecular ion peak at $\mathrm{m} / \mathrm{e} 516,514,496$ and 470 corresponding to $(\mathrm{M}+2)^{++}\left(\mathrm{M}^{+}\right),[\mathrm{M}-\mathrm{H} 2 \mathrm{O}]$, [M-CO2], respectively .

2-(4-Bromophenyl)amino-4-(4-bromophenyl)-4-oxo-butanoic acid (14)

A solution of $1(2.5 \mathrm{~g}, 0.01 \mathrm{~mol})$ in dry benzene $(100 \mathrm{ml})$ was treated with pbromoaniline $((2 \mathrm{ml}), 0.01 \mathrm{~mol})$ and few drops of piperidine and stirred at room temperature for $1 \mathrm{hr}$, then heated under reflux for $3 \mathrm{hr}$. The solid that separated on cooling was crystallized form ethanol. M.wt $=427\left(\mathrm{C}_{16} \mathrm{H}_{13} \mathrm{Br}_{2} \mathrm{NO}_{3}\right.$ ) (m.p $190^{\circ} \mathrm{C}$,

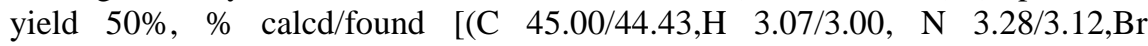
37.47/37.33 IR vNH (vbonded and non bonded), 3227, 2186, C=O1709, $1676 \mathrm{~cm}^{-1}$ and ${ }^{1} \mathrm{HNMR} \delta 3.42(2 \mathrm{dd}, 1 \mathrm{Ha},(\mathrm{J}=15.2, \mathrm{~J}=7.2)$ and $1 \mathrm{Hb}$ methylene protons , $\mathrm{CH}_{2}-\mathrm{C}=\mathrm{O},(\mathrm{J}=15.2, \mathrm{~J}=5.1)$ diastereotopic protons), 4,2 (dd,CH-COO, sterogenic methine proton, $\mathrm{J}=7.2, \mathrm{~J}=5.1), 2 \mathrm{dd}$ at $7.20-7.50$ assigned for $8 \mathrm{ArH}$ aromatic protons, singlet $10.1(\mathrm{~s}, 1 \mathrm{H}, \mathrm{COOH}, \mathrm{a}$ acidic proton which exchanged in $\mathrm{D} 2 \mathrm{O})$.

2-Phthalimidomethyl-4- oxo-5-(4-bromobenzoylmethyl) imidazolo [2,1-b]-1,3,4thiadiazole (15)

A mixture of 13 (3 g;0.005 mol) and acetic anhydride (10ml) was heated under reflux for $1 \mathrm{hr}$. The solid that separated on cooling was crystallized form ethanol. M.wt $=497\left(\mathrm{C}_{21} \mathrm{H}_{13} \mathrm{BrSN}_{4} \mathrm{O}_{4}\right)$ m.p. $230{ }^{\circ} \mathrm{C}$, yield $65 \%$ calcd/found [ $(\mathrm{C} 50$. 89/51.00, H 2.64/2.22, N 11.30/11.62, Br 16.12/16.08, S 6.74/6.38. IR vC=O at $1772,1720,1691,1668 \mathrm{~cm}^{-1}$. ${ }^{1} \mathrm{HNMR}$ DMSO exhibits signals at $3.2(2 \mathrm{dd}$, $\mathrm{CH}_{2-} \mathrm{C}=\mathrm{O} \mathrm{J}=7.7$ ) (diastereotopic protons), 3.9(dd, $\mathrm{CH}-\mathrm{COOH}$, methineproton) 5.2 (s,2H,CH2-N), 6.7(s,1H,bridgeCH, 1,3-double bond shift), 7.2-7.7(m,8H,ArH) . The EI-MS shows the molecular ion peak at m/e 498,496 corresponding to $(\mathrm{M}+2)^{++}\left(\mathrm{M}^{++}\right)$, respectively .

4-([5-Phthalimidomethyl]-1,3,5- thiadiazol-2-yl) amino-6-(4-bromophenyl) 2,3,4,5tetrahydropyridazin-3(2H)-one (16)

A solution of $13(2.15 \mathrm{~g}, 5 \mathrm{mmol})$ and $0.5 \mathrm{ml}$ hydrazine hydrate in $30 \mathrm{ml}$ ethanol was refluxed for $4 \mathrm{hr}$. The solid that separated after cooling was crystallized form the dioxan . M.wt $=511\left(\mathrm{C}_{21} \mathrm{H}_{15} \mathrm{BrSN}_{6} \mathrm{O}_{3}\right)$ m.p. $240{ }^{\circ} \mathrm{C}$,yield $75 \%$ calcd/found [(C49. 32/49.00, H 2.94/2.78, N 16.44/16.72, Br 15.65/15.20 S 6.26/6.22. IR $v \mathrm{NH}, v \mathrm{C}=\mathrm{O}$ at $3400,3310,1770,1691,1680 \mathrm{~cm}^{-1}$. ${ }^{1} \mathrm{HNMR}$ DMSO exhibits signals at $3.2\left(2 \mathrm{dd}, \mathrm{CH}_{2-} \mathrm{C}=\mathrm{O} \mathrm{J}=7.7\right)$ (diastereotopic protons), 4.0(dd, CH-COOH, methineproton) $5.5(\mathrm{~s}, 2 \mathrm{H}, \mathrm{CH} 2), 6.5(\mathrm{~s}, \mathrm{NH}), 7.4-7.8(\mathrm{~m}, 8 \mathrm{H}, \mathrm{ArH})$ $\mathrm{ArH}), 12.2(\mathrm{~s}, 1 \mathrm{H}, \mathrm{NH})$. The EI-MS shows the molecular ion peak at $\mathrm{m} / \mathrm{e} 512$.

4-(4-Bromophenyl)amino-6-(4-bromophenyl) 2,3,4,5-tetrahydropyridazin-3(2H)one (17)

A solution of $14(2.15 \mathrm{~g}, 5 \mathrm{mmol})$ and $0.5 \mathrm{ml}, 0.01$ hydrazine hydrate in $30 \mathrm{ml}$ ethanol was refluxed for $4 \mathrm{hr}$. The solid that separated after cooling was crystallized form the dioxan . M.wt $=423\left(\mathrm{C}_{16} \mathrm{H}_{13} \mathrm{Br}_{2} \mathrm{~N}_{3} \mathrm{O}\right)$ (m.p $206^{\circ} \mathrm{C}$, yield $60 \%$, $\%$ calcd/found [(C 45.42/45.48, H 3.09/3.15,N 9.93/9.98, Br 37.77/37.61. IR 
vOH and/or $v$ NHv $3379,3275,3180$ and $v \mathrm{C}=\mathrm{O} 1676 v \mathrm{C}=\mathrm{N}$ at $1613 \mathrm{~cm}^{-1},{ }^{1} \mathrm{HNMR}$ $\delta 3.22\left(2 \mathrm{dd}, 1 \mathrm{Ha},(\mathrm{J}=15.2, \mathrm{~J}=7.2)\right.$ and $1 \mathrm{Hb}$ methylene protons, $\mathrm{CH}_{2}-\mathrm{C}=\mathrm{O},(\mathrm{J}=15.2$, $\mathrm{J}=6.1$ ) diastereotopic protons), 4,0 (dd, $\mathrm{CH}-\mathrm{COO}$, sterogenic methine proton , $\mathrm{J}=7.2, \mathrm{~J}=6.1), 7.40-7.70(\mathrm{~m}, 8 \mathrm{ArH}$ aromatic protons $), 13.2(\mathrm{~s}, 1 \mathrm{H}, \mathrm{NH}$, a acidic proton which exchanged in D2O). The EI-MS shows the molecular ion peak at $\mathrm{m} / \mathrm{e} 250$ corresponding to (M-4-bromoaniline) $\left.{ }^{+} \quad \mathrm{H}, \mathrm{N}=\right), 2.3$ (dd,2H,CH2 diasteriotopicprotons $\mathrm{J}=14.2, \mathrm{~J}=4.3$ \& $\mathrm{J}=9.4, \mathrm{~J}=4.3), 2.9$ (dd,1H, CHCOO, $\mathrm{J}=14.2, \quad \mathrm{~J}=9.4), \quad 4(\mathrm{q}, 1 \mathrm{H}, \mathrm{CH}-\mathrm{N}, \quad \mathrm{J}=7.5), 7.27 .5 \quad(\mathrm{~m}, 5 \mathrm{H}, \mathrm{ArH}), \quad 8.2(\mathrm{~s}, 1 \mathrm{H}, \mathrm{NH})$, 11(s,1H,COOH). The EI-MS shows the molecular ion peak at m/e 337 and 335 corresponding to $(\mathrm{M}+2)^{++}\left(\mathrm{M}^{++}\right)$, respectively , 510 corresponding to $(\mathrm{M}+2)^{++}$ $\left(\mathrm{M}^{+}\right)$, respectively .

2-Ethoxy carbonyl-4-([5-phthalimidomethyl]-1,3,5-thiadiazol-2-yl)amino -6-(4bromophenyl) 2,3,4,5-tetrahydropyridazin-3(2H)-one (18)

A solution of $16(2.55 \mathrm{~g}, 5 \mathrm{mmol})$ and ethylchloroacetate $(0.9 \mathrm{ml})$ in $30 \mathrm{ml}$ dry acetone in the presence of anhydrous $\mathrm{K} 2 \mathrm{CO} 3$ was heated on water bath for $24 \mathrm{hr}$. The solid that separated after cooling was crystallized form the benzene . M.wt $=597 \quad\left(\mathrm{C}_{25} \mathrm{H}_{21} \mathrm{BrSN}_{6} \mathrm{O}_{5}\right)$ m.p. $120{ }^{\circ} \mathrm{C}$, yield $75 \%$ calcd/found [(C50. 25/50.09, H 3.51/3.23 , N 14.07/14.00 , Br 15.18/14.87 S 5.36/5.22. IR vNH, $v \mathrm{C}=\mathrm{O}$ at $3300,3160,1770,1745,1691,1680 \mathrm{~cm}^{-1} .{ }^{1} \mathrm{HNMR}$ DMSO exhibits signals at $1.5(\mathrm{t}, 3 \mathrm{H}, \mathrm{CH} 3), 3.0\left(2 \mathrm{dd}, \mathrm{CH}_{2-} \mathrm{C}=\mathrm{O} \mathrm{J}=7.7\right)$ (diastereotopic protons), 3.8 (dd,CH-COOH,methineproton), 4.2 (q,2H,OCH2), 5 (s,2H,NCH2CO), 5.5 $(\mathrm{s}, 2 \mathrm{H}, \mathrm{CH} 2), \quad 6.5(\mathrm{~s}, \mathrm{NH}), 7.4-7.8(\mathrm{~m}, 8 \mathrm{H}, \mathrm{ArH}) \quad \mathrm{ArH})$. The EI-MS shows the molecular ion peak at $\mathrm{m} / \mathrm{e} 598,596$ corresponding to $(\mathrm{M}+2)^{.^{+}}\left(\mathrm{M}^{+}\right)$, respectively.

\section{References}

1. Koehler, T., Friedrich, G. and Nmhu, P., Agents Actions, 32 (1-2), 70; C.A.115, 88049z. (1991).

2. Koehler, T., Kirchner, H.M., Peinhard, G., Hirsch-elmann, R. and Nmhu, P., Biochem. Pharmacol. 44 (4), 805, C.A. 117, 204766 Y (1992).

3. Bowden, K., Daplozzo, A. and Duah, C.K., J. Chem. Res. Synap (1990) .

4. Thomas, J.C., U.S. 2, 532-579 (1950): C.A. 45, 1290 (1951).

5. Juranic, Z., Stevovic, L.J., Drakulo, D., Stanojkovic, T. and Radulovic, S.J., Serb. Chem. Soc. 64, 505 (1999).

6. Rizk, S.A., EL-Hashash, M.A. and Aburzeza,M.M., Utility of p-acetamidobenzoyl prop-2enoic acid in the synthesis of new $\alpha$-amino acids and using them as building blocks in heterocyclic synthesis. Egypt. J. Chem. 54 (3), 299-313 (2011).

7. Eissa, A.M.F., J. Chinase, 52, 1205-1214 (2005).

8. El-Hashash, M.A., Abd El-Mageid, G.A., El-Kady, S.S. and Mohammed S.A., 1,4Thioriaalkylation and / or amination of E-3-(4-bromobenzoyl) acrylic acid and some studies with the products. Egypt . J. Chem. 51 (1),103-112 (2008) .

Egypt. J. Chem. 55, No.1 (2012) 
9. Rizk, S.A., El-Hashash, M.A. and Mostafa, K.K., Utility of $\beta$-aroyl acrylic acid in heterocyclic synthesis. Egypt. J. Chem. 51 (5), 116-121 (2008).

10. Elhashash, M., Soliman, A. and Madkour, M., Revue Roumainede Chimie, 38 (8), 955 (1993).

11. El-Hashash, M., Amine, M., Soliman, F. and Morsi, M., J. Serb. Chem. Soc. 57 (9), 563 (1992).

12. Youssef, A., Madkour, H., Marzouk, M., El-Hashash, M. and El-Soll, A.M., Can. J. Chem. 83, 251 (2005) .

13. Rizk, S.A., El-Hashash, M.A. and Aburzeza, M.M., 1,4-Arylation of $\beta$-(4acetylaminobenzoyl) acrylic acid with activated aromatic hydrocarbons under fridelcrafts conditions and some studies with the products. Egypt. J. Chem. 54 (1), 69-87 (2011).

14. Youssef, A.S., Madkour, H.F., Marzouk, M.I., El-Soll, A.M. and El-Hashash, M.A., Utility of 3-Aroyl prop-2-enoic acid in heterocyclic synthesis. Afinidad, 61(512), 304-316 (2003) .

15. Sondhi, S.M., Johar, M., Rajvanshi, S., Dastidar, S.G., Shukla, R., Raghubir, R. and Lown, J.W., Australian J. Chem. 54, 69 (2001) .

16. Ghorab, M. M. and El-Sayed, S. M., Acta Pharm. 49, 149 (1999).

17. Gietma, J.A., Workman, P., Scott, J.E., Crawshaw, A., Dobbis, H.J., Dennis, I., Mulder, N., Sleijfer, D.T.H. and Willemse, P.H.B., Br. J. Cancer. 68, 661 (1993)

18. Nasr, M. N. and Gineinah, M. M., Arch. Pharm. 335, 289 (2002).

19. Cammito, A., Pemmisin, M., Lnu-Due, C., Hoguet, F., Gaultier, C. and Narcisse, J., Eur. J. Chem. 25, 635 (1990).

20. Pemmisin, M., Lue-Due, C., Hoguet, F., Gaultier, C. and Narcisse, J., Eur. J. Chem. 23, 534 (1988).

21. Smith, P.A.S. and Kan, R.O., J. Org. Chem. 29, 2261 (1964).

22. Nega, S., Aionso, J., Diazj, A. and Junquere, F., J. Heterocyclic Chem. 27, 269 (1990).

23. Kuyper, L.F., Baccanari, D.P., Jones, M.L., Hunter, R.N., Tansik, R.L., Joyner, S. S., Boytos, C.M., Rudolph, S.K., Knick, V., Wilson, H. R., Caddell, J.M., Friedman, H. S., Comley J.C.W. and Stables, J.N. , J. Med. Chem. 39, 892 (1996).

24. Hunger, A. and Hoffmann, K., Helv. Chim. Acta, 40, 1319 (1957) .

25. Ismagilva, A.F., Zarudy, F.S., Lazareva, D.N., Davydova, V.A., Karachuria, L. T., Ismagilova, Z.F. and Tropynina, Y.G., Antibiot. Khimioter. 43, 19. Chem. Abst. 129, 76035t (1998) . 
26. Srivastava, V., Negi, A.S., Kumar, J.K., Faridi, U., Sisodia, B.S., Darokar, M.P., Luqman, S. and Khanuja, S.P.S., Bioorg. Med. Chem. Lett. 16, 911 (2006)

27. Toshio, O., Yoshikazu, S., Yoshimi, A., Yasuhiro, I., Tamotsu, F., Noriko, S., Yuji, Y., Tetsuji, A. and Toshikazu, O., J. Antibiot. 53, 337 (2002).

28. Jeffrey, D.P. and Joan, D.A. and Edward, W.J., PCT Int. Appl. 97 (1999) .

29. Piaz, V.D., Vergelli, C., Giovannoni, M.P., Scheideler, M.A., Petrane, G. and Zaratin, P., Farmaco, 58, 1063 (2003).

30. Akahane, A., Katayama, H., Mitsunaga, T., Kato, T., Kinoshita, A., Kita, Y., Kusaunoki, T., Terai,T., Yoshida, K. and Shiokawa, Y., J. Med. Chem. 42, 779 (1999).

31. Horino, H., Mimura, T., Kagechika, K., Ohta, M., Kubo, H. and Kitagawa, M., Chem. Pharm. Bull. 46, 402 (1998).

32. Yamamoto, A., Iwama,T., Takeda, H. and Nagai, H., Jpn. J. Pharmcol. 68, 47 (1995).

33. Tsubaki, K., Taniguchi, K., Tabuchi, S., Okitsu, O., Hahori, K., Soki, J., Sakane, K. and Tanaka, H., Bioorg. Med. Chem. Lett. 12, 2787 (2000).

34. Robert, W. Allcock, Haakon Blakli, Zhong Jiang, Karen A. Johnston, Keith M. Morgan , Georgina M. Rosair , Kazuhiko Iwase, Yasushi Kohno and David R. Adams , Phosphodiesterase inhibitors. Part 1: Synthesis and structure-activity relationships of pyrazolopyridine-pyridazinone PDE inhibitors developed from ibudilast . Bioorganic \& Medicinal Chemistry Letters , 21 , 3307-3312 (2011) . 


\title{
استخدام بعض مشتقات حمض البروبينك في تحضير مركبات غير متجانسة الحلقة مشفة
}

سامح احمد محمد رزق ، ماهر عبد العزيز محمود الحشاش ، سهير احمد شاكر

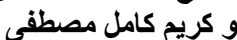

قسم الكيمياء- كلية العلوم- جامعة عين شمس- القاهرة- مصر.

\begin{abstract}
يتضمن هذا البحث 1- تحضير بعض الاحماض البروييك الحاملة مجموعات الاريل

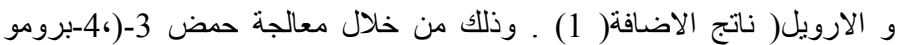

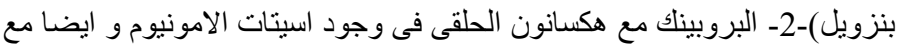

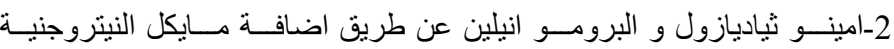

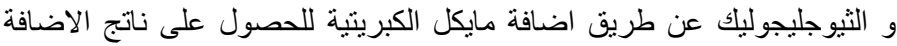
الذى يستخدم لتحضير العديد من المركبات الغير متجانسة الحلقة مثل البيريدازينون

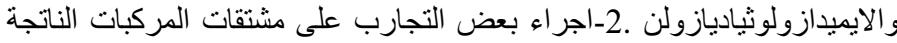

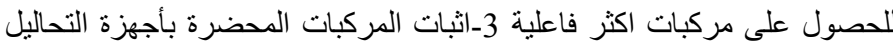
الدقيقة مثل الاشعة تحت الحمر اءو و الرنين المغناطيسى و الكتلة الاكترونى.
\end{abstract}

